\title{
Iterative algorithms for the split variational inequality and fixed point problems under nonlinear transformations
}

\author{
Yonghong Yao ${ }^{a}$, Yeong-Cheng Liou, ${ }^{b, *}$, Jen-Chih $\mathrm{Yao}^{\mathrm{c}}$ \\ ${ }^{a}$ Department of Mathematics, Tianjin Polytechnic University, Tianjin 300387, China. \\ ${ }^{b}$ Department of Healthcare Administration and Medical Informatics; and Research Center of Nonlinear Analysis and Optimization and \\ Center for Fundamental Science, Kaohsiung Medical University, Kaohsiung 807, Taiwan. \\ ${ }^{c}$ Center for General Education, China Medical University, Taichung, 40402, Taiwan.
}

Communicated by Y. J. Cho

\begin{abstract}
In the present paper, we consider the split variational inequality and fixed point problem that requires to find a solution of a generalized variational inequality in a nonempty closed convex subset $\mathcal{C}$ of a real Hilbert space $\mathcal{H}$ whose image under a nonlinear transformation is a fixed point of a pseudocontractive operator. An iterative algorithm is introduced to solve this split problem and the strong convergence analysis is given. (c)2017 All rights reserved.
\end{abstract}

Keywords: Split problem, variational inequality, fixed point, iterative algorithm, pseudocontractive mappings. 2010 MSC: 49J53, 49M37, 65K10, 90C25.

\section{Introduction}

Let $\mathcal{H}$ be a real Hilbert space equipped with inner product $\langle\cdot, \cdot\rangle$ and norm $\|\cdot\|$. The convex feasibility problem can be mathematically formulated as finding a point $p^{b}$ such that

$$
p^{b} \in \bigcap_{m=1}^{k} \mathcal{C}_{m},
$$

where $\left\{\mathcal{C}_{\mathfrak{m}}\right\}_{\mathfrak{m}=1}^{k} \subset \mathcal{H}$ are nonempty closed convex sets. The convex feasibility problem (1.1) has received so much attention due to its extensive applications in many applied disciplines as diverse as approximation theory, image recovery and signal processing, control theory, biomedical engineering, communications, and geophysics. As a special case of the convex feasibility problem, the split feasibility problem can be stated as the following.

Let $\mathcal{H}_{1}$ and $\mathcal{H}_{2}$ be two real Hilbert spaces. Let $\mathcal{C} \subset \mathcal{H}_{1}$ and $\mathcal{Q} \subset \mathcal{H}_{2}$ be two closed convex sets. Let $\Psi: \mathcal{H}_{1} \rightarrow \mathcal{H}_{2}$ be a bounded linear operator. In this setting, the split feasibility problem is to find a point

\footnotetext{
${ }^{*}$ Corresponding author

Email addresses: yaoyonghong@aliyun.com (Yonghong Yao), simplex_liou@hotmail.com (Yeong-Cheng Liou), yaojc@mail.cmu.edu.tw (Jen-Chih Yao)

doi:10.22436/jnsa.010.02.43
} 
$p^{b}$ such that

$$
p^{b} \in \mathcal{C} \text { and } \Psi\left(p^{b}\right) \in \mathcal{Q} .
$$

This problem comes from the intensity-modulated radiation therapy. Since then, a large number of split problems have been introduced and studied extensively. The reader can refer to: Byrne [2], Ceng et al. [3, 4], Censor et al. [5-7], He et al. [10, 11], Qu and Xiu [15], Xu [17, 18], Yang [19], Yao et al. $[21,23,26]$, Zhao [30], and the references therein.

Let $\mathcal{C} \subset \mathcal{H}$ be a nonempty closed convex set. Let $\mathcal{B}: \mathcal{C} \rightarrow \mathcal{H}, \Psi: \mathcal{C} \rightarrow \mathcal{C}$, and $\mathcal{T}: \mathcal{C} \rightarrow \mathcal{C}$ be three nonlinear operators. Recall that the generalized variational inequality (GVI) is to find $u^{b} \in \mathcal{C}$ such that

$$
\left\langle\mathcal{B} u^{b}, \Psi\left(v^{b}\right)-\Psi\left(u^{b}\right)\right\rangle \geqslant 0, \quad \forall v^{b} \in \mathcal{C} .
$$

If $\Psi \equiv \mathcal{J}$, then (1.2) reduces to the variational inequality of finding $\mathrm{u}^{\mathrm{b}} \in \mathcal{C}$ such that

$$
\left\langle\mathcal{B} u^{b}, v^{b}-u^{b}\right\rangle \geqslant 0, \forall v^{b} \in \mathcal{C} .
$$

Variational inequalities have played an import role in the study of several unrelated problems arising in physics, finance, economics, network analysis, elasticity, optimization, water resources, medical images, and structural analysis. For some related work, please refer to: Facchinei and Pang [8], Iusem [12], Glowinski [9], Korpelevich [13], Noor [1], Yao et al. [20, 22, 24, 25, 27], Zegeye et al. [28], and Zhang et al. [29].

In this article, we will study the split variational inequality problem and fixed point problem of finding a point $x^{b}$ such that

$$
p^{b} \in \operatorname{GVI}(\mathcal{B}, \Psi, \mathcal{C}) \text { and } \Psi\left(p^{b}\right) \in \operatorname{Fix}(\mathcal{T}),
$$

where $\operatorname{GVI}(\mathcal{B}, \Psi, \mathcal{C})$ is the solution set of $(1.2)$ and $\operatorname{Fix}(\mathcal{T})$ is the fixed points set of $\mathcal{T}$.

We introduce a new iterative algorithm to solve (1.3). We prove the strong convergence of the presented algorithm under some mild conditions.

\section{Preliminaries}

In this section, we give several definitions and lemmas.

Definition 2.1. An operator $\mathcal{T}: \mathcal{C} \rightarrow \mathcal{C}$ is said to be L-Lipschitz continuous if

$$
\left\|\mathcal{T} u^{\dagger}-\mathcal{T} v^{\dagger}\right\| \leqslant \mathrm{L}\left\|\mathrm{u}^{\dagger}-v^{\dagger}\right\|, \quad \forall \mathrm{u}^{\dagger}, v^{\dagger} \in \mathcal{C},
$$

where $L$ is a positive constant.

Definition 2.2. An operator $\mathcal{T}: \mathcal{C} \rightarrow \mathcal{C}$ is called pseudocontractive if

$$
\left\langle\mathcal{T} u^{\dagger}-\mathcal{T} v^{\dagger}, u^{\dagger}-v^{\dagger}\right\rangle \leqslant\left\|u^{\dagger}-v^{\dagger}\right\|^{2}, \quad \forall u^{\dagger}, v^{\dagger} \in \mathcal{C},
$$

or

$$
\left\|\mathcal{T} u^{\dagger}-\mathcal{T} v^{\dagger}\right\|^{2} \leqslant\left\|u^{\dagger}-v^{\dagger}\right\|^{2}+\left\|(\mathcal{J}-\mathcal{T}) u^{\dagger}-(\mathcal{J}-\mathcal{T}) v^{\dagger}\right\|^{2}, \quad \forall u^{\dagger}, v^{\dagger} \in \mathcal{C} .
$$

Definition 2.3. An operator $\mathcal{B}: \mathcal{C} \rightarrow \mathcal{H}$ is said to be

- monotone if

$$
\left\langle u^{b}-v^{b}, \mathcal{B} u^{b}-\mathcal{B} v^{b}\right\rangle \geqslant 0, \forall u^{b}, v^{b} \in \mathcal{C} .
$$

- strongly monotone if

$$
\left\langle\mathrm{u}^{b}-v^{b}, \mathcal{B} u^{b}-\mathcal{B} v^{b}\right\rangle \geqslant \delta\left\|u^{b}-v^{b}\right\|^{2}, \quad \forall u^{b}, v^{b} \in \mathcal{C},
$$

where $\delta$ is a positive constant. 
- inverse strongly monotone if

$$
\left\langle u^{b}-v^{b}, \mathcal{B} u^{b}-\mathcal{B} v^{b}\right\rangle \geqslant \alpha\left\|\mathcal{B} u^{b}-\mathcal{B} v^{b}\right\|^{2}, \forall u^{b}, v^{b} \in \mathcal{C},
$$

where $\alpha$ is a positive constant.

- $\alpha$-inverse strongly $\Psi$-monotone if

$$
\left\langle\Psi\left(u^{\dagger}\right)-\Psi\left(v^{\dagger}\right), \mathcal{B} u^{b}-\mathcal{B} v^{b}\right\rangle \geqslant \alpha\left\|\mathcal{B} u^{b}-\mathcal{B} v^{b}\right\|^{2}, \forall u^{b}, v^{b} \in \mathcal{C},
$$

where $\Psi: \mathcal{C} \rightarrow \mathcal{C}$ is a nonlinear operator and $\alpha$ is a positive constant.

Let $\mathcal{A}: \mathcal{H} \rightarrow 2^{\mathcal{H}}$ be a set-valued operator. The effective domain of $\mathcal{A}$ is denoted by $\operatorname{dom}(\mathcal{A})$, i.e., $\operatorname{dom}(\mathcal{A})=\{x \in \mathcal{H}: \mathcal{A} x \neq \emptyset\}$. A multi-valued operator $\mathcal{A}$ is said to be a monotone on $\mathcal{H}$ iff $\langle x-y, u-v\rangle \geqslant 0$ for all $x, y \in \operatorname{dom}(\mathcal{A}), u \in \mathcal{A x}$, and $v \in \mathcal{A} y$. A monotone operator $\mathcal{A}$ on $\mathcal{H}$ is said to be maximal iff its graph is not strictly contained in the graph of any other monotone operator on $\mathcal{H}$.

For any $u^{b} \in \mathcal{H}$, there exists a unique nearest point in $\mathcal{C}$, denoted by $\operatorname{proj}_{\mathcal{C}}\left[\mathfrak{u}^{b}\right]$ satisfying

$$
\left\|u^{b}-\operatorname{proj}_{\mathcal{C}}\left[u^{b}\right]\right\| \leqslant\left\|u^{b}-u^{\dagger}\right\|
$$

for all $u^{\dagger} \in \mathcal{C}$.

The operator $\operatorname{proj}_{\mathcal{C}}: \mathcal{H} \rightarrow \mathcal{C}$ is firmly nonexpansive. Especially,

$$
\left\langle u^{b}-\operatorname{proj}_{\mathcal{C}}\left[u^{b}\right], u^{\dagger}-\operatorname{proj}_{\mathcal{C}}\left[u^{b}\right]\right\rangle \leqslant 0, \quad \forall u^{b} \in \mathcal{H}, u^{\dagger} \in \mathcal{C} .
$$

We call the operator $\mathcal{T}$ is demiclosed if the following relationship holds

$$
u_{\mathfrak{n}} \rightarrow \tilde{u} \text { and } \mathcal{T} u_{\mathfrak{n}} \rightarrow \mathfrak{u} \text { imply } \mathcal{T}(\tilde{\mathfrak{u}})=u .
$$

Lemma 2.4 ([23]). Let $\mathcal{H}$ be a Hilbert space. Let $\mathcal{T}: \mathcal{H} \rightarrow \mathcal{H}$ be a $\mathrm{k}$-Lipschitz pseudocontractive operator. Then,

$$
\left\|(1-\sigma) u^{\sharp}+\sigma \mathcal{T}\left((1-\delta) u^{\sharp}+\delta \mathcal{T} u^{\sharp}\right)-u^{b}\right\|^{2} \leqslant\left\|u^{\sharp}-u^{b}\right\|^{2}+\sigma(\sigma-\delta)\left\|\mathcal{T}\left((1-\delta) u^{\sharp}+\delta \mathcal{T} u^{\sharp}\right)-u^{\sharp}\right\|^{2},
$$

for all $\mathrm{u}^{\sharp} \in \mathcal{H}$ and $\mathrm{u}^{b} \in \operatorname{Fix}(\mathcal{T})$, when $0<\sigma<\delta<\frac{1}{\sqrt{1+\kappa^{2}}+1}$.

Lemma 2.5 ([23]). Let $\mathcal{H}$ be a Hilbert space. Let $\mathcal{T}: \mathcal{H} \rightarrow \mathcal{H}$ be a $\mathrm{k}$-Lipschitzian operator with $\mathrm{\kappa} \geqslant 1$. Then,

$$
\operatorname{Fix}(((1-v) \mathcal{J}+v \mathcal{T}) \mathcal{T})=\operatorname{Fix}(\mathcal{T}((1-v) \mathcal{J}+v \mathcal{T}))=\operatorname{Fix}(\mathcal{T}),
$$

for all $v \in\left(0, \frac{1}{\mathrm{~K}}\right)$.

Lemma 2.6 ([31]). Let $\mathcal{H}$ be a real Hilbert space, and $\mathcal{C}$ a closed convex subset of $\mathcal{H}$. Let $\mathcal{T}: \mathcal{C} \rightarrow \mathcal{C}$ be a continuous pseudocontractive operator. Then $\mathcal{J}-\mathcal{T}$ is demi-closed at zero.

Lemma 2.7 ([23]). Let $\mathcal{H}$ be a Hilbert space. Let $\mathcal{T}: \mathcal{H} \rightarrow \mathcal{H}$ be a $\mathrm{k}$-Lipschitzian operator with $\mathrm{\kappa} \geqslant 1$. If $\mathcal{J}-\mathcal{T}$ is demiclosed at 0 , then $\mathcal{J}-\mathcal{T}((1-v) \mathcal{J}+v \mathcal{T})$ is also demiclosed at 0 when $v \in\left(0, \frac{1}{k}\right)$.

Lemma 2.8 ([16]). Let $\left\{\varpi_{n}\right\} \subset[0, \infty),\left\{\sigma_{n}\right\} \subset(0,1)$, and $\left\{\rho_{n}\right\}$ be three sequences such that

$$
\varpi_{n+1} \leqslant\left(1-\sigma_{n}\right) \varpi_{n}+\rho_{n}, \forall n \geqslant 1 .
$$

Assume the following restrictions are satisfied

(i) $\sum_{n=1}^{\infty} \sigma_{n}=\infty$;

(ii) $\limsup _{n \rightarrow \infty} \frac{\rho_{n}}{\sigma_{n}} \leqslant 0$ or $\sum_{n=1}^{\infty}\left|\rho_{n}\right|<\infty$.

Then, $\lim _{n \rightarrow \infty} \varpi_{n}=0$.

Lemma 2.9 ([14]). Let $\left\{w_{n}\right\}$ be a sequence of real numbers. Assume $\left\{w_{n}\right\}$ does not decrease at infinity, that is, there exists at least a subsequence $\left\{w_{n_{k}}\right\}$ of $\left\{w_{n}\right\}$ such that $w_{n_{k}} \leqslant w_{n_{k}+1}$ for all $k \geqslant 0$. For every $n \geqslant N_{0}$, define an integer sequence $\{\tau(\mathrm{n})\}$ as

$$
\tau(n)=\max \left\{i \leqslant n: w_{n_{i}}<w_{n_{i}+1}\right\} .
$$

Then, $\tau(\mathrm{n}) \rightarrow \infty$ as $\mathrm{n} \rightarrow \infty$ and for all $\mathrm{n} \geqslant \mathrm{N}_{0}$

$$
\max \left\{w_{\tau(n)}, w_{n}\right\} \leqslant w_{\tau(n)+1} .
$$




\section{Main results}

In this section, we consider the following split problem.

Problem 3.1. Let $\mathcal{C}$ be a nonempty closed convex subset of a real Hilbert space $\mathcal{H}$. Let $\Psi: \mathcal{C} \rightarrow \mathcal{C}$ be a weakly continuous and $\delta$-strongly monotone mapping such that its $\operatorname{rang} \mathrm{R}(\Psi)=\mathcal{C}$. Let $\mathcal{B}: \mathcal{C} \rightarrow \mathcal{H}$ be an $\alpha$-inverse strongly $\Psi$-monotone mapping. Let $\mathcal{T}: \mathcal{C} \rightarrow \mathcal{C}$ be a $\kappa$-Lipschitzian pseudocontractive operator with $k \geqslant 1$.

Our objective is to

$$
\text { find } \chi^{\ddagger} \in \operatorname{GVI}(\mathcal{B}, \Psi, \mathcal{C}) \text { such that } \Psi\left(\chi^{\ddagger}\right) \in \operatorname{Fix}(\mathcal{T}) \text {. }
$$

Denote the solution set of (3.1) by $S$, that is, $S=\operatorname{GVI}(\mathcal{B}, \Psi, \mathcal{C}) \cap \Psi^{-1}(\operatorname{Fix}(\mathcal{T}))$. In the sequel, we assume $S \neq \emptyset$. We firstly present our algorithm for solving the split problem (3.1).

Algorithm 3.2. For given initial value $x_{0} \in \mathcal{C}$ arbitrarily, let the sequence $\left\{x_{n}\right\}$ be generated iteratively by

$$
\left\{\begin{array}{l}
u_{n}=\operatorname{proj}_{\mathcal{e}}\left[\alpha_{n} \sigma \phi\left(x_{n}\right)+\left(1-\alpha_{n}\right)\left(\Psi\left(x_{n}\right)-\gamma_{n} \mathcal{B} x_{n}\right)\right], \\
\Psi\left(x_{n+1}\right)=\vartheta_{n} \Psi\left(x_{n}\right)+\left(1-\vartheta_{n}\right)\left[\left(1-\sigma_{n}\right) u_{n}+\sigma_{n} \mathcal{T}\left(\left(1-\delta_{n}\right) u_{n}+\delta_{n} \mathcal{T} u_{n}\right)\right], n \geqslant 0,
\end{array}\right.
$$

where $\phi: \mathcal{C} \rightarrow \mathcal{H}$ is an L-Lipschitz continuous operator, $\left\{\alpha_{n}\right\},\left\{\vartheta_{n}\right\},\left\{\sigma_{n}\right\}$, and $\left\{\delta_{n}\right\}$ are four real number sequences in $[0,1],\left\{\gamma_{n}\right\}$ is a real number sequence in $(0, \infty)$, and $\sigma>0$ is a constant.

Theorem 3.3. Assume the following conditions are satisfied:

(C1) $\lim _{n \rightarrow \infty} \alpha_{n}=0$ and $\sum_{n} \alpha_{n}=\infty$;

(C2) $0<\liminf _{n \rightarrow \infty} \vartheta_{n} \leqslant \limsup \sup _{n \rightarrow \infty} \vartheta_{n}<1$ and $0<a<\sigma_{n}<c<\delta_{n}<b<\frac{1}{\sqrt{1+k^{2}}+1}$;

(C3) $0<\liminf _{n \rightarrow \infty} \gamma_{n} \leqslant \limsup _{n \rightarrow \infty} \gamma_{n}<2 \alpha$ and $\delta \in(L \sigma, 2 \alpha)$.

Then, the sequence $\left\{x_{n}\right\}$ generated by (3.2) converges strongly to $x^{\ddagger} \in S$ which solves the following variational inequality

$$
\left\langle\sigma \phi\left(x^{\ddagger}\right)-\Psi\left(x^{\ddagger}\right), \Psi\left(x^{b}\right)-\Psi\left(x^{\ddagger}\right)\right\rangle \leqslant 0, \quad \forall x^{b} \in S .
$$

Proof. According to the strong monotonicity of $\Psi$, we have

$$
\delta\|x-y\|^{2} \leqslant\langle\Psi(x)-\Psi(y), x-y\rangle \leqslant\|\Psi(x)-\Psi(y)\|\|x-y\|, \quad \forall x, y \in \mathcal{C} .
$$

Thus,

$$
\|\Psi(x)-\Psi(y)\| \geqslant \delta\|x-y\|, \quad \forall x, y \in \mathcal{C} .
$$

Now, we show that the solution of the variational inequality (3.3) is unique. Assume that both $x^{*}$ and $\tilde{x}$ solve (3.3). Then, we obtain

$$
\left\langle\sigma \phi\left(x^{*}\right)-\Psi\left(x^{*}\right), \Psi(\tilde{x})-\Psi\left(x^{*}\right)\right\rangle \leqslant 0 \text { and }\left\langle\sigma \phi(\tilde{x})-\Psi(\tilde{x}), \Psi\left(x^{*}\right)-\Psi(\tilde{x})\right\rangle \leqslant 0 .
$$

Hence,

$$
\left\langle\sigma \phi(\tilde{x})-\Psi(\tilde{x})-\sigma \phi\left(\chi^{*}\right)+\Psi\left(\chi^{*}\right), \Psi\left(x^{*}\right)-\Psi(\tilde{x})\right\rangle \leqslant 0 .
$$

It follows that

$$
\left\|\Psi\left(x^{*}\right)-\Psi(\tilde{x})\right\|^{2} \leqslant \sigma\left\langle\phi\left(x^{*}\right)-\phi(\tilde{x}), \Psi\left(x^{*}\right)-\Psi(\tilde{x})\right\rangle \leqslant \sigma\left\|\phi\left(x^{*}\right)-\phi(\tilde{x})\right\|\left\|\Psi\left(x^{*}\right)-\Psi(\tilde{x})\right\| .
$$

This together with (3.4) implies that

$$
\delta\left\|x^{*}-\tilde{x}\right\| \leqslant\left\|\Psi\left(x^{*}\right)-\Psi(\tilde{x})\right\| \leqslant \sigma\left\|\phi\left(x^{*}\right)-\phi(\tilde{x})\right\| \leqslant \sigma L\left\|x^{*}-\tilde{x}\right\| .
$$

Since $\sigma \mathrm{L}<\delta$, by the condition (C3), we deduce $x^{*}=\tilde{\chi}$ immediately. So, the variational inequality (3.3) has a unique solution denoted by $\chi^{\ddagger}$. Thus, $\chi^{\ddagger} \in \operatorname{GVI}(\mathcal{B}, \Psi, \mathcal{C})$ and $\Psi\left(\chi^{\ddagger}\right) \in \operatorname{Fix}(\mathcal{T})$. By $(2.1)$, we have 
$\Psi\left(\chi^{\ddagger}\right)=\operatorname{proj}_{\mathcal{C}}\left[\Psi\left(\chi^{\ddagger}\right)-\gamma_{n} \mathcal{B} \chi^{\ddagger}\right]$ for all $n \geqslant 0$. Since $\mathcal{B}$ is $\alpha$-inverse strongly $\Psi$-monotone, we derive

$$
\begin{aligned}
\|(\Psi(x) & -\gamma \mathcal{B} x)-\left(\Psi\left(x^{\ddagger}\right)-\gamma \mathcal{B} x^{\ddagger}\right) \|^{2} \\
& =\left\|\Psi(x)-\Psi\left(x^{\ddagger}\right)\right\|^{2}-2 \gamma\left\langle\mathcal{B} x-\mathcal{B} x^{\ddagger}, \Psi(x)-\Psi\left(x^{\ddagger}\right)\right\rangle+\gamma^{2}\left\|\mathcal{B} x-\mathcal{B} x^{\ddagger}\right\|^{2} \\
& \leqslant\left\|\Psi(x)-\Psi\left(x^{\ddagger}\right)\right\|^{2}-2 \gamma \alpha\left\|\mathcal{B} x-\mathcal{B} x^{\ddagger}\right\|^{2}+\gamma^{2}\left\|\mathcal{B} x-\mathcal{B} x^{\ddagger}\right\|^{2} \\
& \leqslant\left\|\Psi(x)-\Psi\left(x^{\ddagger}\right)\right\|^{2}+\gamma(\gamma-2 \alpha)\left\|\mathcal{B} x-\mathcal{B} x^{\ddagger}\right\|^{2} .
\end{aligned}
$$

Applying (3.5), we get

$$
\begin{aligned}
\left\|\left(\Psi\left(x_{n}\right)-\gamma_{n} \mathcal{B} x_{n}\right)-\left(\Psi\left(\chi^{\ddagger}\right)-\gamma_{n} \mathcal{B} x^{\ddagger}\right)\right\|^{2} & \leqslant\left\|\Psi\left(x_{n}\right)-\Psi\left(\chi^{\ddagger}\right)\right\|^{2}+\gamma_{n}\left(\gamma_{n}-2 \alpha\right)\left\|\mathcal{B} x_{n}-\mathcal{B} x^{\ddagger}\right\|^{2} \\
& \leqslant\left\|\Psi\left(x_{n}\right)-\Psi\left(\chi^{\ddagger}\right)\right\|^{2}
\end{aligned}
$$

and

$$
\begin{aligned}
\| \Psi\left(x_{n+1}\right) & -\gamma_{n+1} \mathcal{B} x_{n+1}-\left(\Psi\left(x_{n}\right)-\gamma_{n+1} \mathcal{B} x_{n}\right) \|^{2} \\
& \leqslant\left\|\Psi\left(x_{n+1}\right)-\Psi\left(x_{n}\right)\right\|^{2}+\gamma_{n+1}\left(\gamma_{n+1}-2 \alpha\right)\left\|\mathcal{B} x_{n+1}-\mathcal{B} x_{n}\right\|^{2} .
\end{aligned}
$$

From (3.2), (3.4), and (3.6), we have

$$
\begin{aligned}
\left\|u_{n}-\Psi\left(x^{\ddagger}\right)\right\|= & \left\|\operatorname{proj}_{\mathcal{C}}\left[\alpha_{n} \sigma \phi\left(x_{n}\right)+\left(1-\alpha_{n}\right)\left(\Psi\left(x_{n}\right)-\gamma_{n} \mathcal{B} x_{n}\right)\right]-\operatorname{proj}_{\mathcal{C}}\left[\Psi\left(x^{\ddagger}\right)-\gamma_{n} \mathcal{B} x^{\ddagger}\right]\right\| \\
\leqslant & \left\|\alpha_{n}\left(\sigma \phi\left(x_{n}\right)-\Psi\left(x^{\ddagger}\right)+\gamma_{n} \mathcal{B} x^{\ddagger}\right)+\left(1-\alpha_{n}\right)\left(\left(\Psi\left(x_{n}\right)-\gamma_{n} \mathcal{B} x_{n}\right)-\left(\Psi\left(x^{\ddagger}\right)-\gamma_{n} \mathcal{B} x^{\ddagger}\right)\right)\right\| \\
\leqslant & \alpha_{n}\left\|\sigma \phi\left(x_{n}\right)-\sigma \phi\left(x^{\ddagger}\right)\right\|+\alpha_{n}\left\|\sigma \phi\left(x^{\ddagger}\right)-\Psi\left(x^{\ddagger}\right)+\gamma_{n} \mathcal{B} x^{\ddagger}\right\| \\
& +\left(1-\alpha_{n}\right)\left\|\left(\Psi\left(x_{n}\right)-\gamma_{n} \mathcal{B} x_{n}\right)-\left(\Psi\left(x^{\ddagger}\right)-\gamma_{n} \mathcal{B} x^{\ddagger}\right)\right\| \\
\leqslant & \alpha_{n} \sigma \mathrm{L}\left\|x_{n}-x^{\ddagger}\right\|+\alpha_{n}\left\|\sigma \phi\left(x^{\ddagger}\right)-\Psi\left(x^{\ddagger}\right)+\gamma_{n} \mathcal{B} x^{\ddagger}\right\|+\left(1-\alpha_{n}\right)\left\|\Psi\left(x_{n}\right)-\Psi\left(x^{\ddagger}\right)\right\| \\
\leqslant & \alpha_{n} \sigma \mathrm{L} / \delta\left\|\Psi\left(x_{n}\right)-\Psi\left(x^{\ddagger}\right)\right\|+\alpha_{n}\left\|\sigma \phi\left(x^{\ddagger}\right)-\Psi\left(x^{\ddagger}\right)+\gamma_{n} \mathcal{B} x^{\ddagger}\right\| \\
& +\left(1-\alpha_{n}\right)\left\|\Psi\left(x_{n}\right)-\Psi\left(x^{\ddagger}\right)\right\| \\
= & {\left[1-(1-\sigma \mathrm{L} / \delta) \alpha_{n}\right]\left\|\Psi\left(x_{n}\right)-\Psi\left(x^{\ddagger}\right)\right\|+\alpha_{n}\left\|\sigma \phi\left(x^{\ddagger}\right)-\Psi\left(x^{\ddagger}\right)+\gamma_{n} \mathcal{B} x^{\ddagger}\right\| } \\
\leqslant & {\left[1-(1-\sigma \mathrm{L} / \delta) \alpha_{n}\right]\left\|\Psi\left(x_{n}\right)-\Psi\left(x^{\ddagger}\right)\right\|+\alpha_{n}\left(\left\|\sigma \phi\left(x^{\ddagger}\right)-\Psi\left(x^{\ddagger}\right)\right\|+2 \alpha\left\|\mathcal{B} x^{\ddagger}\right\|\right) . }
\end{aligned}
$$

By (3.6) and (3.7), we obtain

$$
\begin{aligned}
\| u_{n} & -\Psi\left(x^{\ddagger}\right) \|^{2} \\
& \leqslant\left\|\alpha_{n}\left(\sigma \phi\left(x_{n}\right)-\Psi\left(x^{\ddagger}\right)+\gamma_{n} \mathcal{B} x^{\ddagger}\right)+\left(1-\alpha_{n}\right)\left(\left(\Psi\left(x_{n}\right)-\gamma_{n} \mathcal{B} x_{n}\right)-\left(\Psi\left(x^{\ddagger}\right)-\gamma_{n} \mathcal{B} x^{\ddagger}\right)\right)\right\|^{2} \\
& \left.\leqslant \alpha_{n}\left\|\sigma \phi\left(x_{n}\right)-\Psi\left(x^{\ddagger}\right)+\gamma_{n} \mathcal{B} x^{\ddagger}\right\|^{2}+\left(1-\alpha_{n}\right) \|\left(\Psi\left(x_{n}\right)-\gamma_{n} \mathcal{B} x_{n}\right)-\left(\Psi\left(x^{\ddagger}\right)-\gamma_{n} \mathcal{B} x^{\ddagger}\right)\right) \|^{2} \\
& \leqslant \alpha_{n}\left\|\sigma \phi\left(x_{n}\right)-\Psi\left(x^{\ddagger}\right)+\gamma_{n} \mathcal{B} x^{\ddagger}\right\|^{2}+\left(1-\alpha_{n}\right)\left[\left\|\Psi\left(x_{n}\right)-\Psi\left(x^{\ddagger}\right)\right\|^{2}+\gamma_{n}\left(\gamma_{n}-2 \alpha\right)\left\|\mathcal{B} x_{n}-\mathcal{B} x^{\ddagger}\right\|^{2}\right] .
\end{aligned}
$$

Set $y_{n}=\left(1-\sigma_{n}\right) u_{n}+\sigma_{n} \mathcal{T}\left(\left(1-\delta_{n}\right) u_{n}+\delta_{n} \mathcal{T} u_{n}\right)$ for all $n \geqslant 0$. In view of Lemma 2.4, we deduce

$$
\begin{aligned}
\left\|y_{n}-\Psi\left(\chi^{\ddagger}\right)\right\|^{2} & =\left\|\left(1-\sigma_{n}\right) u_{n}+\sigma_{n} \mathcal{T}\left(\left(1-\delta_{n}\right) u_{n}+\delta_{n} \mathcal{T} u_{n}\right)-\Psi\left(\chi^{\ddagger}\right)\right\|^{2} \\
& \leqslant\left\|u_{n}-\Psi\left(\chi^{\ddagger}\right)\right\|^{2}+\sigma_{n}\left(\sigma_{n}-\delta_{n}\right)\left\|\mathcal{T}\left(\left(1-\delta_{n}\right) u_{n}+\delta_{n} \mathcal{T} u_{n}\right)-u_{n}\right\|^{2} \\
& \leqslant\left\|u_{n}-\Psi\left(x^{\ddagger}\right)\right\|^{2} .
\end{aligned}
$$

From (3.2), (3.7), and (3.9), we have

$$
\begin{aligned}
\left\|\Psi\left(x_{n+1}\right)-\Psi\left(x^{\ddagger}\right)\right\| \leqslant & \vartheta_{n}\left\|\Psi\left(x_{n}\right)-\Psi\left(x^{\ddagger}\right)\right\|+\left(1-\vartheta_{n}\right)\left\|y_{n}-\Psi\left(x^{\ddagger}\right)\right\| \\
\leqslant & \vartheta_{n}\left\|\Psi\left(x_{n}\right)-\Psi\left(x^{\ddagger}\right)\right\|+\left(1-\vartheta_{n}\right)\left\|u_{n}-\Psi\left(x^{\ddagger}\right)\right\| \\
\leqslant & \vartheta_{n}\left\|\Psi\left(x_{n}\right)-\Psi\left(x^{\ddagger}\right)\right\|+\left(1-\vartheta_{n}\right)\left[1-(1-\sigma \mathrm{L} / \delta) \alpha_{n}\right]\left\|\Psi\left(x_{n}\right)-\Psi\left(x^{\ddagger}\right)\right\| \\
& +\left(1-\vartheta_{n}\right) \alpha_{n}\left(\left\|\sigma \phi\left(x^{\ddagger}\right)-\Psi\left(x^{\ddagger}\right)\right\|+2 \alpha\left\|\mathcal{B} x^{\ddagger}\right\|\right) \\
= & {\left[1-(1-\sigma \mathrm{L} / \delta)\left(1-\vartheta_{n}\right) \alpha_{n}\right]\left\|\Psi\left(x_{n}\right)-\Psi\left(x^{\ddagger}\right)\right\| } \\
& +(1-\sigma \mathrm{L} / \delta)\left(1-\vartheta_{n}\right) \alpha_{n} \frac{\left\|\sigma \phi\left(x^{\ddagger}\right)-\Psi\left(x^{\ddagger}\right)\right\|+2 \alpha\left\|\mathcal{B} \chi^{\ddagger}\right\|}{1-\sigma \mathrm{L} / \delta} .
\end{aligned}
$$


By induction

$$
\left\|\Psi\left(x_{n}\right)-\Psi\left(x^{\ddagger}\right)\right\| \leqslant \max \left\{\left\|\Psi\left(x_{0}\right)-\Psi\left(x^{\ddagger}\right)\right\|, \frac{\left\|\sigma \phi\left(x^{\ddagger}\right)-\Psi\left(x^{\ddagger}\right)\right\|+2 \alpha\left\|\mathcal{B} \chi^{\ddagger}\right\|}{1-\sigma L / \delta}\right\} .
$$

It follows that

$$
\left\|x_{n}-\chi^{\ddagger}\right\| \leqslant \frac{1}{\delta}\left\|\Psi\left(x_{n}\right)-\Psi\left(\chi^{\ddagger}\right)\right\| \leqslant \frac{1}{\delta} \max \left\{\left\|\Psi\left(x_{0}\right)-\Psi\left(\chi^{\ddagger}\right)\right\|, \frac{\left\|\sigma \phi\left(x^{\ddagger}\right)-\Psi\left(x^{\ddagger}\right)\right\|+2 \alpha\left\|\mathcal{B} \chi^{\ddagger}\right\|}{1-\sigma \mathrm{L} / \delta}\right\} .
$$

Hence, $\left\{\Psi\left(x_{n}\right)\right\}$ and $\left\{x_{n}\right\}$ are all bounded. From (3.2), we have

$$
\Psi\left(x_{n+1}\right)-\Psi\left(x_{n}\right)=\left(1-\vartheta_{n}\right)\left(y_{n}-\Psi\left(x_{n}\right)\right) .
$$

Hence,

$$
\left\langle\Psi\left(x_{n+1}\right)-\Psi\left(x_{n}\right), \Psi\left(x_{n}\right)-\Psi\left(x^{\ddagger}\right)\right\rangle=\left(1-\vartheta_{n}\right)\left\langle y_{n}-\Psi\left(x_{n}\right), \Psi\left(x_{n}\right)-\Psi\left(x^{\ddagger}\right)\right\rangle .
$$

Note that

$$
\begin{aligned}
2\left\langle\Psi\left(x_{n+1}\right)-\Psi\left(x_{n}\right), \Psi\left(x_{n}\right)-\Psi\left(x^{\ddagger}\right)\right\rangle= & \left\|\Psi\left(x_{n+1}\right)-\Psi\left(x^{\ddagger}\right)\right\|^{2}-\left\|\Psi\left(x_{n}\right)-\Psi\left(\chi^{\ddagger}\right)\right\|^{2} \\
& -\left\|\Psi\left(x_{n+1}\right)-\Psi\left(x_{n}\right)\right\|^{2},
\end{aligned}
$$

and

$$
2\left\langle y_{n}-\Psi\left(x_{n}\right), \Psi\left(x_{n}\right)-\Psi\left(x^{\ddagger}\right)\right\rangle=\left\|y_{n}-\Psi\left(x^{\ddagger}\right)\right\|^{2}-\left\|\Psi\left(x_{n}\right)-\Psi\left(x^{\ddagger}\right)\right\|^{2}-\left\|y_{n}-\Psi\left(x_{n}\right)\right\|^{2} .
$$

By virtue of (3.12), (3.13), and (3.14), we deduce

$$
\begin{aligned}
\| \Psi\left(x_{n+1}\right) & -\Psi\left(x^{\ddagger}\right)\left\|^{2}-\right\| \Psi\left(x_{n}\right)-\Psi\left(x^{\ddagger}\right)\left\|^{2}-\right\| \Psi\left(x_{n+1}\right)-\Psi\left(x_{n}\right) \|^{2} \\
& =\left(1-\vartheta_{n}\right)\left[\left\|y_{n}-\Psi\left(x^{\ddagger}\right)\right\|^{2}-\left\|\Psi\left(x_{n}\right)-\Psi\left(x^{\ddagger}\right)\right\|^{2}-\left\|y_{n}-\Psi\left(x_{n}\right)\right\|^{2}\right] .
\end{aligned}
$$

Combining (3.11) with (3.15), we have

$$
\begin{aligned}
\| \Psi\left(x_{n+1}\right)- & \Psi\left(x^{\ddagger}\right)\left\|^{2}-\right\| \Psi\left(x_{n}\right)-\Psi\left(x^{\ddagger}\right) \|^{2} \\
= & \left(1-\vartheta_{n}\right)\left[\left\|y_{n}-\Psi\left(x^{\ddagger}\right)\right\|^{2}-\left\|\Psi\left(x_{n}\right)-\Psi\left(x^{\ddagger}\right)\right\|^{2}-\left\|y_{n}-\Psi\left(x_{n}\right)\right\|^{2}\right] \\
& +\left(1-\vartheta_{n}\right)^{2}\left\|y_{n}-\Psi\left(x_{n}\right)\right\|^{2} \\
= & \left(1-\vartheta_{n}\right)\left[\left\|y_{n}-\Psi\left(x^{\ddagger}\right)\right\|^{2}-\left\|\Psi\left(x_{n}\right)-\Psi\left(x^{\ddagger}\right)\right\|^{2}\right]-\vartheta_{n}\left(1-\vartheta_{n}\right)\left\|y_{n}-\Psi\left(x_{n}\right)\right\|^{2} \\
\leqslant & \left(1-\vartheta_{n}\right)\left[\left\|u_{n}-\Psi\left(x^{\ddagger}\right)\right\|^{2}-\left\|\Psi\left(x_{n}\right)-\Psi\left(x^{\ddagger}\right)\right\|^{2}\right]-\vartheta_{n}\left(1-\vartheta_{n}\right)\left\|y_{n}-\Psi\left(x_{n}\right)\right\|^{2} .
\end{aligned}
$$

Returning to (3.10), we get

$$
\begin{aligned}
\left\|u_{n}-\Psi\left(x^{\ddagger}\right)\right\|^{2} \leqslant & {\left[1-(1-\sigma \mathrm{L} / \delta) \alpha_{n}\right]\left\|\Psi\left(x_{n}\right)-\Psi\left(x^{\ddagger}\right)\right\|^{2} } \\
& +(1-\sigma \mathrm{L} / \delta) \alpha_{n}\left(\frac{\left\|\sigma \phi\left(x^{\ddagger}\right)-\Psi\left(x^{\ddagger}\right)\right\|+2 \alpha\left\|\mathcal{B} \chi^{\ddagger}\right\|}{(1-\sigma L / \delta)}\right)^{2} .
\end{aligned}
$$

Next, we consider two possible cases.

Case 1. Assume there exist some integer $m>0$ such that $\left\{\left\|\Psi\left(x_{n}\right)-\Psi\left(x^{\ddagger}\right)\right\|\right\}$ is decreasing for all $n \geqslant m$.

In this case, we know that $\lim _{n \rightarrow \infty}\left\|\Psi\left(x_{n}\right)-\Psi\left(x^{\ddagger}\right)\right\|$ exists. From (3.16) and (3.17), we have

$$
\begin{aligned}
\vartheta_{n}\left(1-\vartheta_{n}\right)\left\|y_{n}-\Psi\left(x_{n}\right)\right\|^{2} \leqslant & \left\|\Psi\left(x_{n}\right)-\Psi\left(x^{\ddagger}\right)\right\|^{2}-\left\|\Psi\left(x_{n+1}\right)-\Psi\left(x^{\ddagger}\right)\right\|^{2} \\
& +\left(1-\vartheta_{n}\right)\left[\left\|u_{n}-\Psi\left(x^{\ddagger}\right)\right\|^{2}-\left\|\Psi\left(x_{n}\right)-\Psi\left(x^{\ddagger}\right)\right\|^{2}\right] \\
\leqslant & \left\|\Psi\left(x_{n}\right)-\Psi\left(x^{\ddagger}\right)\right\|^{2}-\left\|\Psi\left(x_{n+1}\right)-\Psi\left(x^{\ddagger}\right)\right\|^{2} \\
& +(1-\sigma L / \delta) \alpha_{n}\left(\frac{\left\|\sigma \phi\left(x^{\ddagger}\right)-\Psi\left(x^{\ddagger}\right)\right\|+2 \alpha\left\|\mathcal{B} \chi^{\ddagger}\right\|}{(1-\sigma L / \delta)}\right)^{2} \\
& \rightarrow 0 .
\end{aligned}
$$


This together with (C2) implies that

$$
\lim _{n \rightarrow \infty}\left\|y_{n}-\Psi\left(x_{n}\right)\right\|=0 .
$$

Furthermore, it follows from (3.11) that

$$
\lim _{n \rightarrow \infty}\left\|\Psi\left(x_{n+1}\right)-\Psi\left(x_{n}\right)\right\|=0 .
$$

By (3.8), we have

$$
\begin{aligned}
\left\|\Psi\left(x_{n+1}\right)-\Psi\left(x^{\ddagger}\right)\right\|^{2}= & \left\|\vartheta_{n}\left(\Psi\left(x_{n}\right)-\Psi\left(x^{\ddagger}\right)\right)+\left(1-\vartheta_{n}\right)\left(y_{n}-\Psi\left(x^{\ddagger}\right)\right)\right\|^{2} \\
\leqslant & \vartheta_{n}\left\|\Psi\left(x_{n}\right)-\Psi\left(x^{\ddagger}\right)\right\|^{2}+\left(1-\vartheta_{n}\right)\left\|y_{n}-\Psi\left(x^{\ddagger}\right)\right\|^{2} \\
\leqslant & \vartheta_{n}\left\|\Psi\left(x_{n}\right)-\Psi\left(x^{\ddagger}\right)\right\|^{2}+\left(1-\vartheta_{n}\right)\left\|u_{n}-\Psi\left(x^{\ddagger}\right)\right\|^{2} \\
\leqslant & \left(1-\vartheta_{n}\right) \alpha_{n}\left\|\sigma \phi\left(x_{n}\right)-\Psi\left(x^{\ddagger}\right)+\gamma_{n} \mathcal{B} x^{\ddagger}\right\|^{2}+\vartheta_{n}\left\|\Psi\left(x_{n}\right)-\Psi\left(x^{\ddagger}\right)\right\|^{2} \\
& +\left(1-\vartheta_{n}\right)\left(1-\alpha_{n}\right)\left\|\Psi\left(x_{n}\right)-\Psi\left(x^{\ddagger}\right)\right\|^{2} \\
& \left.+\left(1-\vartheta_{n}\right)\left(1-\alpha_{n}\right) \gamma_{n}\left(\gamma_{n}-2 \alpha\right)\left\|\mathcal{B} x_{n}-\mathcal{B} x^{\ddagger}\right\|^{2}\right] \\
\leqslant & \left\|\Psi\left(x_{n}\right)-\Psi\left(x^{\ddagger}\right)\right\|^{2}+\left(1-\vartheta_{n}\right) \alpha_{n}\left\|\sigma \phi\left(x_{n}\right)-\Psi\left(x^{\ddagger}\right)+\gamma_{n} \mathcal{B} x^{\ddagger}\right\|^{2} \\
& +\left(1-\vartheta_{n}\right)\left(1-\alpha_{n}\right) \gamma_{n}\left(\gamma_{n}-2 \alpha\right)\left\|\mathcal{B} x_{n}-\mathcal{B} x^{\ddagger}\right\|^{2} .
\end{aligned}
$$

Thus,

$$
\begin{aligned}
& \left(1-\vartheta_{n}\right)\left(1-\alpha_{n}\right) \gamma_{n}\left(2 \alpha-\gamma_{n}\right)\left\|\mathcal{B} x_{n}-\mathcal{B} x^{\ddagger}\right\|^{2} \\
& \quad \leqslant \\
& \quad \leqslant \Psi\left(x_{n}\right)-\Psi\left(x^{\ddagger}\right)\left\|^{2}-\right\| \Psi\left(x_{n+1}\right)-\Psi\left(x^{\ddagger}\right)\left\|^{2}+\left(1-\vartheta_{n}\right) \alpha_{n}\right\| \sigma \phi\left(x_{n}\right)-\Psi\left(x^{\ddagger}\right)+\gamma_{n} \mathcal{B} x^{\ddagger} \|^{2} \\
& \left.\quad \leqslant\left(x_{n}\right)-\Psi\left(x^{\ddagger}\right)\|+\| \Psi\left(x_{n+1}\right)-\Psi\left(x^{\ddagger}\right) \|\right)\left\|\Psi\left(x_{n+1}\right)-\Psi\left(x_{n}\right)\right\| \\
& \quad+\left(1-\vartheta_{n}\right) \alpha_{n}\left\|\sigma \phi\left(x_{n}\right)-\Psi\left(x^{\ddagger}\right)+\gamma_{n} \mathcal{B} x^{\ddagger}\right\|^{2} \\
& \quad \rightarrow 0 \text { (by (C1) and (3.19)). }
\end{aligned}
$$

Since $\liminf _{n \rightarrow \infty}\left(1-\vartheta_{n}\right)\left(1-\alpha_{n}\right) \gamma_{n}\left(2 \alpha-\gamma_{n}\right)>0$, we obtain

$$
\lim _{n \rightarrow \infty}\left\|\mathcal{B} x_{n}-\mathcal{B} x^{\ddagger}\right\|=0 .
$$

Set $z_{n}=\Psi\left(x_{n}\right)-\gamma_{n} \mathcal{B} x_{n}-\left(\Psi\left(x^{\ddagger}\right)-\gamma_{n} \mathcal{B} x^{\ddagger}\right)$ for all $n$. Using (2.1), we get

$$
\begin{aligned}
\left\|u_{n}-\Psi\left(x^{\ddagger}\right)\right\|^{2}= & \| \operatorname{proj}_{\mathcal{C}}\left[\alpha_{n} \sigma \phi\left(x_{n}\right)+\left(1-\alpha_{n}\right)\left(\Psi\left(x_{n}\right)-\gamma_{n} \mathcal{B} x_{n}\right)\right]-\operatorname{proj}_{\mathcal{C}}\left[\Psi\left(x^{\ddagger}\right)-\gamma_{n} \mathcal{B} x^{\ddagger} \|^{2}\right. \\
\leqslant & \left\langle\alpha_{n}\left(\sigma \phi\left(x_{n}\right)-\Psi\left(x^{\ddagger}\right)+\gamma_{n} \mathcal{B} x^{\ddagger}\right)+\left(1-\alpha_{n}\right) z_{n}, u_{n}-\Psi\left(x^{\ddagger}\right)\right\rangle \\
= & \frac{1}{2}\left\{\left\|\alpha_{n}\left(\sigma \phi\left(x_{n}\right)-\Psi\left(x^{\ddagger}\right)+\gamma_{n} \mathcal{B} x^{\ddagger}\right)+\left(1-\alpha_{n}\right) z_{n}\right\|^{2}+\left\|u_{n}-\Psi\left(x^{\ddagger}\right)\right\|^{2}\right. \\
& \left.-\left\|\alpha_{n}\left(\sigma \phi\left(x_{n}\right)-\Psi\left(x^{\ddagger}\right)+\gamma_{n} \mathcal{B} x^{\ddagger}\right)+\left(1-\alpha_{n}\right) z_{n}-u_{n}+\Psi\left(x^{\ddagger}\right)\right\|^{2}\right\} \\
\leqslant & \frac{1}{2}\left\{\alpha_{n}\left\|\sigma \phi\left(x_{n}\right)-\Psi\left(x^{\ddagger}\right)+\gamma_{n} \mathcal{B} x^{\ddagger}\right\|^{2}+\left(1-\alpha_{n}\right)\left\|\Psi\left(x_{n}\right)-\Psi\left(x^{\ddagger}\right)\right\|^{2}\right. \\
& +\left\|u_{n}-\Psi\left(x^{\ddagger}\right)\right\|^{2}-\| \alpha_{n}\left(\sigma \phi\left(x_{n}\right)-\Psi\left(x^{\ddagger}\right)+\gamma_{n} \mathcal{B} x^{\ddagger}-z_{n}\right)+\Psi\left(x_{n}\right) \\
& \left.-u_{n}-\gamma_{n}\left(\mathcal{B} x_{n}-\mathcal{B} x^{\ddagger}\right) \|^{2}\right\} \\
= & \frac{1}{2}\left\{\alpha_{n}\left\|\sigma \phi\left(x_{n}\right)-\Psi\left(x^{\ddagger}\right)+\gamma_{n} \mathcal{B} x^{\ddagger}\right\|^{2}+\left(1-\alpha_{n}\right)\left\|\Psi\left(x_{n}\right)-\Psi\left(x^{\ddagger}\right)\right\|^{2}\right. \\
& +\left\|u_{n}-\Psi\left(x^{\ddagger}\right)\right\|^{2}-\left\|\Psi\left(x_{n}\right)-u_{n}\right\|^{2}-\alpha_{n}^{2}\left\|\sigma \phi\left(x_{n}\right)-\Psi\left(x^{\ddagger}\right)+\gamma_{n} \mathcal{B} x^{\ddagger}-z_{n}\right\|^{2} \\
& -\gamma_{n}^{2}\left\|\mathcal{B} x_{n}-\mathcal{B} x^{\ddagger}\right\|+2 \gamma_{n} \alpha_{n}\left\langle\mathcal{B} x_{n}-\mathcal{B} x^{\ddagger}, \sigma \phi\left(x_{n}\right)-\Psi\left(x^{\ddagger}\right)+\gamma_{n} \mathcal{B} x^{\ddagger}-z_{n}\right\rangle \\
& +2 \gamma_{n}\left\langle\Psi\left(x_{n}\right)-u_{n}, \mathcal{B} x_{n}-\mathcal{B} x^{\ddagger}\right\rangle \\
& \left.-2 \alpha_{n}\left\langle\Psi\left(x_{n}\right)-u_{n}, \sigma \phi\left(x_{n}\right)-\Psi\left(x^{\ddagger}\right)+\gamma_{n} \mathcal{B} x^{\ddagger}-z_{n}\right\rangle\right\} .
\end{aligned}
$$


It follows that

$$
\begin{aligned}
\left\|u_{n}-\Psi\left(x^{\ddagger}\right)\right\|^{2} \leqslant & \alpha_{n}\left\|\sigma \phi\left(x_{n}\right)-\Psi\left(x^{\ddagger}\right)+\gamma_{n} \mathcal{B} x^{\ddagger}\right\|^{2}+\left(1-\alpha_{n}\right)\left\|\Psi\left(x_{n}\right)-\Psi\left(x^{\ddagger}\right)\right\|^{2} \\
& -\left\|\Psi\left(x_{n}\right)-u_{n}\right\|^{2}+2 \gamma_{n}\left\|\Psi\left(x_{n}\right)-u_{n}\right\|\left\|\mathcal{B} x_{n}-\mathcal{B} x^{\ddagger}\right\| \\
& +2 \alpha_{n}\left\|\Psi\left(x_{n}\right)-u_{n}\right\|\left\|\sigma \phi\left(x_{n}\right)-\Psi\left(x^{\ddagger}\right)+\gamma_{n} \mathcal{B} x^{\ddagger}-z_{n}\right\| \\
& +2 \gamma_{n} \alpha_{n}\left\|\mathcal{B} x_{n}-\mathcal{B} x^{\ddagger}\right\|\left\|\sigma \phi\left(x_{n}\right)-\Psi\left(x^{\ddagger}\right)+\gamma_{n} \mathcal{B} x^{\ddagger}-z_{n}\right\| .
\end{aligned}
$$

In the light of (3.20) and (3.22), we have

$$
\begin{aligned}
\left\|\Psi\left(x_{n+1}\right)-\Psi\left(x^{\ddagger}\right)\right\|^{2} \leqslant & \vartheta_{n}\left\|\Psi\left(x_{n}\right)-\Psi\left(x^{\ddagger}\right)\right\|^{2}+\left(1-\vartheta_{n}\right)\left\|u_{n}-\Psi\left(x^{\ddagger}\right)\right\|^{2} \\
\leqslant & \vartheta_{n}\left\|\Psi\left(x_{n}\right)-\Psi\left(x^{\ddagger}\right)\right\|^{2}+\left(1-\vartheta_{n}\right) \alpha_{n}\left\|\sigma \phi\left(x_{n}\right)-\Psi\left(x^{\ddagger}\right)+\gamma_{n} \mathcal{B} x^{\ddagger}\right\|^{2} \\
& +\left(1-\alpha_{n}\right)\left(1-\vartheta_{n}\right)\left\|\Psi\left(x_{n}\right)-\Psi\left(x^{\ddagger}\right)\right\|^{2}-\left(1-\vartheta_{n}\right)\left\|\Psi\left(x_{n}\right)-u_{n}\right\|^{2} \\
& +2 \gamma_{n}\left(1-\vartheta_{n}\right) \alpha_{n}\left\|\mathcal{B} x_{n}-\mathcal{B} x^{\ddagger}\right\|\left\|\sigma \phi\left(x_{n}\right)-\Psi\left(x^{\ddagger}\right)+\gamma_{n} \mathcal{B} x^{\ddagger}-z_{n}\right\| \\
& +2 \gamma_{n}\left(1-\vartheta_{n}\right)\left\|\Psi\left(x_{n}\right)-u_{n}\right\|\left\|\mathcal{B} x_{n}-\mathcal{B} x^{\ddagger}\right\| \\
& +2\left(1-\vartheta_{n}\right) \alpha_{n}\left\|\Psi\left(x_{n}\right)-u_{n}\right\|\left\|\sigma \phi\left(x_{n}\right)-\Psi\left(x^{\ddagger}\right)+\gamma_{n} \mathcal{B} x^{\ddagger}-z_{n}\right\| \\
\leqslant & \left\|\Psi\left(x_{n}\right)-\Psi\left(x^{\ddagger}\right)\right\|^{2}+\alpha_{n}\left\|\sigma \phi\left(x_{n}\right)-\Psi\left(x^{\ddagger}\right)+\gamma_{n} \mathcal{B} x^{\ddagger}\right\|^{2} \\
& +2 \gamma_{n} \alpha_{n}\left\|\mathcal{B} x_{n}-\mathcal{B} x^{\ddagger}\right\|\left\|\sigma \phi\left(x_{n}\right)-\Psi\left(x^{\ddagger}\right)+\gamma_{n} \mathcal{B} x^{\ddagger}-z_{n}\right\| \\
& +2 \gamma_{n}\left\|\Psi\left(x_{n}\right)-u_{n}\right\|\left\|\mathcal{B} x_{n}-\mathcal{B} x^{\ddagger}\right\|-\left(1-\vartheta_{n}\right)\left\|\Psi\left(x_{n}\right)-u_{n}\right\|^{2} \\
& +2 \alpha_{n}\left\|\Psi\left(x_{n}\right)-u_{n}\right\|\left\|\sigma \phi\left(x_{n}\right)-\Psi\left(x^{\ddagger}\right)+\gamma_{n} \mathcal{B} x^{\ddagger}-z_{n}\right\| .
\end{aligned}
$$

Then,

$$
\begin{aligned}
\left(1-\vartheta_{n}\right)\left\|\Psi\left(x_{n}\right)-u_{n}\right\|^{2} \leqslant & \left(\left\|\Psi\left(x_{n}\right)-\Psi\left(x^{\ddagger}\right)\right\|+\left\|\Psi\left(x_{n+1}\right)-\Psi\left(x^{\ddagger}\right)\right\|\right)\left\|\Psi\left(x_{n+1}\right)-\Psi\left(x_{n}\right)\right\| \\
& +\alpha_{n}\left\|\sigma \phi\left(x_{n}\right)-\Psi\left(x^{\ddagger}\right)+\gamma_{n} \mathcal{B} x^{\ddagger}\right\|^{2} \\
& +2 \gamma_{n} \alpha_{n}\left\|\mathcal{B} x_{n}-\mathcal{B} x^{\ddagger}\right\|\left\|\sigma \phi\left(x_{n}\right)-\Psi\left(x^{\ddagger}\right)+\gamma_{n} \mathcal{B} x^{\ddagger}-z_{n}\right\| \\
& +2 \gamma_{n}\left\|\Psi\left(x_{n}\right)-u_{n}\right\|\left\|\mathcal{B} x_{n}-\mathcal{B} x^{\ddagger}\right\| \\
& +2 \alpha_{n}\left\|\Psi\left(x_{n}\right)-u_{n}\right\|\left\|\sigma \phi\left(x_{n}\right)-\Psi\left(x^{\ddagger}\right)+\gamma_{n} \mathcal{B} x^{\ddagger}-z_{n}\right\| .
\end{aligned}
$$

According to (C1), (3.19), and (3.21), we easily get

$$
\lim _{n \rightarrow \infty}\left\|\Psi\left(x_{n}\right)-u_{n}\right\|=0
$$

Next, we prove $\limsup _{n \rightarrow \infty}\left\langle\sigma \phi\left(\chi^{\ddagger}\right)-\Psi\left(\chi^{\ddagger}\right), u_{n}-\Psi\left(\chi^{\ddagger}\right)\right\rangle \leqslant 0$. Pick up a subsequence $\left\{u_{n_{i}}\right\}$ of $\left\{u_{n}\right\}$ such that

$$
\begin{aligned}
\limsup _{n \rightarrow \infty}\left\langle\sigma \phi\left(x^{\ddagger}\right)-\Psi\left(x^{\ddagger}\right), u_{n}-\Psi\left(x^{\ddagger}\right)\right\rangle & =\lim _{i \rightarrow \infty}\left\langle\sigma \phi\left(x^{\ddagger}\right)-\Psi\left(x^{\ddagger}\right), u_{n_{i}}-\Psi\left(x^{\ddagger}\right)\right\rangle \\
& =\lim _{i \rightarrow \infty}\left\langle\sigma \phi\left(x^{\ddagger}\right)-\Psi\left(x^{\ddagger}\right), \Psi\left(x_{n_{i}}\right)-\Psi\left(x^{\ddagger}\right)\right\rangle .
\end{aligned}
$$

Since $\left\{x_{n_{i}}\right\}$ is bounded, there exists a subsequence $\left\{x_{n_{i_{j}}}\right\}$ of $\left\{x_{n_{i}}\right\}$ which converges weakly to some point $z \in \mathcal{C}$. Without loss of generality, we may assume that $x_{n_{i}} \rightarrow z$. This implies that $\Psi\left(x_{n_{i}}\right) \rightarrow \Psi(z)$ due to the weak continuity of $\Psi$. Thus, $u_{n_{i}} \rightarrow \Psi(z)$ by (3.23).

In view of (3.9), we get

$$
\begin{aligned}
\sigma_{n}\left(\delta_{n}-\sigma_{n}\right)\left\|\mathcal{T}\left(\left(1-\delta_{n}\right) u_{n}+\delta_{n} \mathcal{T} u_{n}\right)-u_{n}\right\|^{2} & \leqslant\left\|u_{n}-\Psi\left(\chi^{\ddagger}\right)\right\|^{2}-\left\|y_{n}-\Psi\left(\chi^{\ddagger}\right)\right\|^{2} \\
& \leqslant\left\|u_{n}-y_{n}\right\|\left(\left\|u_{n}-\Psi\left(x^{\ddagger}\right)\right\|+\left\|y_{n}-\Psi\left(x^{\ddagger}\right)\right\|\right) .
\end{aligned}
$$

It follows from (3.18), (3.23), and (3.25) that

$$
\lim _{n \rightarrow \infty}\left\|\mathcal{T}\left(\left(1-\delta_{n}\right) u_{n}+\delta_{n} \mathcal{T} u_{n}\right)-u_{n}\right\|=0 .
$$


Apply Lemmas 2.5, 2.6, and 2.7 to (3.26) to deduce $\Psi(z) \in \operatorname{Fix}(\mathcal{T})$. Next, we need to prove $z \in \operatorname{GVI}(\mathcal{B}, \Psi, \mathcal{C})$. Set

$$
\mathrm{R} v= \begin{cases}\mathcal{B} v+\mathrm{N}_{\mathcal{C}}(v), & v \in \mathcal{C}, \\ \emptyset, & v \notin \mathcal{C} .\end{cases}
$$

By [29], we know that $R$ is maximal $\Psi$-monotone. Let $(v, w) \in G(R)$. Since $w-\mathcal{B} v \in N_{\mathcal{C}}(v)$ and $x_{n} \in \mathcal{C}$, we have $\left\langle\Psi(v)-\Psi\left(x_{n}\right), w-\mathcal{B} v\right\rangle \geqslant 0$. Noting that $u_{n}=\operatorname{proj}_{\mathcal{C}}\left[\alpha_{n} \sigma \phi\left(x_{n}\right)+\left(1-\alpha_{n}\right)\left(\Psi\left(x_{n}\right)-\gamma_{n} \mathcal{B} x_{n}\right)\right]$, we get

$$
\left\langle\Psi(v)-u_{n}, u_{n}-\left[\alpha_{n} \sigma \phi\left(x_{n}\right)+\left(1-\alpha_{n}\right)\left(\Psi\left(x_{n}\right)-\gamma_{n} \mathcal{B} x_{n}\right)\right]\right\rangle \geqslant 0 .
$$

It follows that

$$
\left\langle\Psi(v)-u_{n}, \frac{u_{n}-\Psi\left(x_{n}\right)}{\gamma_{n}}+\mathcal{B} x_{n}-\frac{\alpha_{n}}{\gamma_{n}}\left(\sigma \phi\left(x_{n}\right)-\Psi\left(x_{n}\right)+\gamma_{n} \mathcal{B} x_{n}\right)\right\rangle \geqslant 0
$$

Then,

$$
\begin{aligned}
\left\langle\Psi(v)-\Psi\left(x_{n_{i}}\right), w\right\rangle \geqslant & \left\langle\Psi(v)-\Psi\left(x_{n_{i}}\right), \mathcal{B} v\right\rangle \\
\geqslant & \left\langle\Psi(v)-\Psi\left(x_{n_{i}}\right), \mathcal{B} v\right\rangle-\left\langle\Psi(v)-u_{n_{i}}, \frac{u_{n_{i}}-\Psi\left(x_{n_{i}}\right)}{\gamma_{n_{i}}}\right\rangle \\
& -\left\langle\Psi(v)-u_{n_{i}}, \mathcal{B} x_{n_{i}}\right\rangle+\frac{\alpha_{n_{i}}}{\gamma_{n_{i}}}\left\langle\Psi(v)-u_{n_{i}}, \sigma \phi\left(x_{n_{i}}\right)-\Psi\left(x_{n_{i}}\right)+\gamma_{n_{i}} \mathcal{B} x_{n_{i}}\right\rangle \\
= & \left\langle\Psi(v)-\Psi\left(x_{n_{i}}\right), \mathcal{B} v-\mathcal{B} x_{n_{i}}\right\rangle+\left\langle\Psi(v)-\Psi\left(x_{n_{i}}\right), \mathcal{B} x_{n_{i}}\right\rangle \\
& -\left\langle\Psi(v)-u_{n_{i}}, \frac{u_{n_{i}}-\Psi\left(x_{n_{i}}\right)}{\gamma_{n_{i}}}\right\rangle-\left\langle\Psi(v)-u_{n_{i}}, \mathcal{B} x_{n_{i}}\right\rangle \\
& +\frac{\alpha_{n_{i}}}{\gamma_{n_{i}}}\left\langle\Psi(v)-u_{n_{i}}, \sigma \phi\left(x_{n_{i}}\right)-\Psi\left(x_{n_{i}}\right)+\gamma_{n_{i}} \mathcal{B} x_{n_{i}}\right\rangle \\
\geqslant & -\left\langle\Psi(v)-u_{n_{i}}, \frac{u_{n_{i}}-\Psi\left(x_{n_{i}}\right)}{\gamma_{n_{i}}}\right\rangle-\left\langle\Psi\left(x_{n_{i}}\right)-u_{n_{i}}, \mathcal{B} x_{n_{i}}\right\rangle \\
& +\frac{\alpha_{n_{i}}}{\gamma_{n_{i}}}\left\langle\Psi(v)-u_{n_{i}}, \sigma \phi\left(x_{n_{i}}\right)-\Psi\left(x_{n_{i}}\right)+\gamma_{n_{i}} \mathcal{B} x_{n_{i}}\right\rangle .
\end{aligned}
$$

Since $\left\|\Psi\left(x_{n_{i}}\right)-u_{n_{i}}\right\| \rightarrow 0$ and $\Psi\left(x_{n_{i}}\right) \rightarrow \Psi(z)$, we deduce that $\langle\Psi(v)-\Psi(z), w\rangle \geqslant 0$ by taking $i \rightarrow \infty$ in (3.27). Thus, $z \in R^{-1} 0$ by the maximal $\Psi$-monotonicity of $R$. Hence, $z \in \operatorname{GVI}(\mathcal{B}, \Psi, \mathcal{C})$. Therefore, $z \in S$.

From (3.24), we obtain

$$
\begin{aligned}
\limsup _{n \rightarrow \infty}\left\langle\sigma \phi\left(x^{\ddagger}\right)-\Psi\left(x^{\ddagger}\right), u_{n}-\Psi\left(x^{\ddagger}\right)\right\rangle & =\lim _{i \rightarrow \infty}\left\langle\sigma \phi\left(x^{\ddagger}\right)-\Psi\left(x^{\ddagger}\right), \Psi\left(x_{n_{i}}\right)-\Psi\left(x^{\ddagger}\right)\right\rangle \\
& =\left\langle\sigma \phi\left(x^{\ddagger}\right)-\Psi\left(\chi^{\ddagger}\right), \Psi(z)-\Psi\left(x^{\ddagger}\right)\right\rangle \leqslant 0 .
\end{aligned}
$$

Note that

$$
\begin{aligned}
\left\|u_{n}-\Psi\left(x^{\ddagger}\right)\right\|^{2}= & \left\|\operatorname{proj}_{\mathcal{C}}\left[\alpha_{n} \sigma \phi\left(x_{n}\right)+\left(1-\alpha_{n}\right)\left(\Psi\left(x_{n}\right)-\gamma_{n} \mathcal{B} x_{n}\right)\right]-\operatorname{proj}_{\mathcal{C}}\left[\Psi\left(x^{\ddagger}\right)-\left(1-\alpha_{n}\right) \gamma_{n} \mathcal{B} x^{\ddagger}\right]\right\|^{2} \\
\leqslant & \left\langle\alpha_{n}\left(\sigma \phi\left(x_{n}\right)-\Psi\left(x^{\ddagger}\right)\right)+\left(1-\alpha_{n}\right) z_{n}, u_{n}-\Psi\left(x^{\ddagger}\right)\right\rangle \\
\leqslant & \alpha_{n} \sigma\left\langle\phi\left(x_{n}\right)-\phi\left(x^{\ddagger}\right), u_{n}-\Psi\left(x^{\ddagger}\right)\right\rangle+\alpha_{n}\left\langle\sigma \phi\left(x^{\ddagger}\right)-\Psi\left(x^{\ddagger}\right), u_{n}-\Psi\left(x^{\ddagger}\right)\right\rangle \\
& +\left(1-\alpha_{n}\right)\left\|\Psi\left(x_{n}\right)-\gamma_{n} \mathcal{B} x_{n}-\left(\Psi\left(x^{\ddagger}\right)-\gamma_{n} \mathcal{B} x^{\ddagger}\right)\right\|\left\|u_{n}-\Psi\left(x^{\ddagger}\right)\right\| \\
\leqslant & \alpha_{n} L \sigma\left\|x_{n}-x^{\ddagger}\right\|\left\|u_{n}-\Psi\left(x^{\ddagger}\right)\right\|+\alpha_{n}\left\langle\sigma \phi\left(x^{\ddagger}\right)-\Psi\left(x^{\ddagger}\right), u_{n}-\Psi\left(x^{\ddagger}\right)\right\rangle \\
& +\left(1-\alpha_{n}\right)\left\|\Psi\left(x_{n}\right)-\Psi\left(x^{\ddagger}\right)\right\|\left\|u_{n}-\Psi\left(x^{\ddagger}\right)\right\| \\
\leqslant & \alpha_{n}(\sigma L / \delta)\left\|\Psi\left(x_{n}\right)-\Psi\left(x^{\ddagger}\right)\right\|\left\|u_{n}-\Psi\left(x^{\ddagger}\right)\right\|+\alpha_{n}\left\langle\sigma \phi\left(x^{\ddagger}\right)-\Psi\left(x^{\ddagger}\right), u_{n}-\Psi\left(x^{\ddagger}\right)\right\rangle \\
& +\left(1-\alpha_{n}\right)\left\|\Psi\left(x_{n}\right)-\Psi\left(x^{\ddagger}\right)\right\|\left\|u_{n}-\Psi\left(x^{\ddagger}\right)\right\| \\
= & {\left[1-(1-\operatorname{Lo} / \delta) \alpha_{n}\right]\left\|\Psi\left(x_{n}\right)-\Psi\left(x^{\ddagger}\right)\right\|\left\|u_{n}-\Psi\left(x^{\ddagger}\right)\right\|+\alpha_{n}\left\langle\sigma \phi\left(x^{\ddagger}\right)-\Psi\left(x^{\ddagger}\right), u_{n}-\Psi\left(x^{\ddagger}\right)\right\rangle } \\
\leqslant & \frac{1-(1-\operatorname{L\sigma } / \delta) \alpha_{n}}{2}\left\|\Psi\left(x_{n}\right)-\Psi\left(x^{\ddagger}\right)\right\|^{2}+\frac{1}{2}\left\|u_{n}-\Psi\left(x^{\ddagger}\right)\right\|^{2}+\alpha_{n}\left\langle\sigma \phi\left(x^{\ddagger}\right)-\Psi\left(x^{\ddagger}\right), u_{n}-\Psi\left(x^{\ddagger}\right)\right\rangle .
\end{aligned}
$$


It follows that

$$
\left\|u_{n}-\Psi\left(x^{\ddagger}\right)\right\|^{2} \leqslant\left[1-(1-L \sigma / \delta) \alpha_{n}\right]\left\|\Psi\left(x_{n}\right)-\Psi\left(x^{\ddagger}\right)\right\|^{2}+2 \alpha_{n}\left\langle\sigma \phi\left(\chi^{\ddagger}\right)-\Psi\left(x^{\ddagger}\right), u_{n}-\Psi\left(x^{\ddagger}\right)\right\rangle .
$$

Therefore,

$$
\begin{aligned}
\left\|\Psi\left(x_{n+1}\right)-\Psi\left(x^{\ddagger}\right)\right\|^{2} \leqslant & \vartheta_{n}\left\|\Psi\left(x_{n}\right)-\Psi\left(x^{\ddagger}\right)\right\|^{2}+\left(1-\vartheta_{n}\right)\left\|u_{n}-\Psi\left(x^{\ddagger}\right)\right\|^{2} \\
\leqslant & \vartheta_{n}\left\|\Psi\left(x_{n}\right)-\Psi\left(x^{\ddagger}\right)\right\|^{2}+\left(1-\vartheta_{n}\right)\left[1-(1-\sigma \mathrm{L} / \delta) \alpha_{n}\right]\left\|\Psi\left(x_{n}\right)-\Psi\left(x^{\ddagger}\right)\right\|^{2} \\
& +2\left(1-\vartheta_{n}\right) \alpha_{n}\left\langle\sigma \phi\left(x^{\ddagger}\right)-\Psi\left(x^{\ddagger}\right), u_{n}-\Psi\left(x^{\ddagger}\right)\right\rangle \\
= & {\left[1-(1-\sigma \mathrm{L} / \delta)\left(1-\vartheta_{n}\right) \alpha_{n}\right]\left\|\Psi\left(x_{n}\right)-\Psi\left(x^{\ddagger}\right)\right\|^{2} } \\
& +2\left(1-\vartheta_{n}\right) \alpha_{n}\left\langle\sigma \phi\left(x^{\ddagger}\right)-\Psi\left(x^{\ddagger}\right), u_{n}-\Psi\left(x^{\ddagger}\right)\right\rangle \\
= & {\left[1-(1-\sigma \mathrm{L} / \delta)\left(1-\vartheta_{n}\right) \alpha_{n}\right]\left\|\Psi\left(x_{n}\right)-\Psi\left(x^{\ddagger}\right)\right\|^{2} } \\
& +(1-\sigma \mathrm{L} / \delta)\left(1-\vartheta_{n}\right) \alpha_{n}\left(\frac{2}{1-\sigma \mathrm{L} / \delta}\left\langle\sigma \phi\left(x^{\ddagger}\right)-\Psi\left(x^{\ddagger}\right), u_{n}-\Psi\left(x^{\ddagger}\right)\right\rangle\right) .
\end{aligned}
$$

We can therefore apply Lemma 2.8 to (3.29) to conclude that $\Psi\left(x_{n}\right) \rightarrow \Psi\left(x^{\ddagger}\right)$ and $x_{n} \rightarrow x^{\ddagger}$.

Case 2. Assume there exists an integer $n_{0}$ such that $\left\|\Psi\left(x_{n_{0}}\right)-\Psi\left(x^{\ddagger}\right)\right\| \leqslant\left\|\Psi\left(x_{n_{0}+1}\right)-\Psi\left(x^{\ddagger}\right)\right\|$. At this case, we set $\omega_{n}=\left\{\left\|\Psi\left(x_{n}\right)-\Psi\left(x^{\ddagger}\right)\right\|\right\}$. Then, we have $\omega_{n_{0}} \leqslant \omega_{n_{0}+1}$. Define an integer sequence $\left\{\tau_{n}\right\}$ for all $n \geqslant n_{0}$ as follows:

$$
\tau(n)=\max \left\{l \in \mathbb{N} \mid n_{0} \leqslant l \leqslant n, \omega_{l} \leqslant \omega_{l+1}\right\} .
$$

It is clear that $\tau(n)$ is a non-decreasing sequence satisfying

$$
\lim _{n \rightarrow \infty} \tau(n)=\infty \text { and } \omega_{\tau(n)} \leqslant \omega_{\tau(n)+1}
$$

for all $n \geqslant n_{0}$.

By the similar argument as that of (3.28) and (3.29), we can prove that

$$
\limsup _{n \rightarrow \infty}\left\langle\sigma \phi\left(\chi^{\ddagger}\right)-\Psi\left(\chi^{\ddagger}\right), u_{\tau(n)}-\Psi\left(\chi^{\ddagger}\right)\right\rangle \leqslant 0
$$

and

$$
\begin{aligned}
\omega_{\tau(n)+1}^{2} \leqslant & {\left[1-(1-\sigma \mathrm{L} / \delta)\left(1-\vartheta_{\tau(n)}\right) \alpha_{\tau(n)}\right] \omega_{\tau(n)}^{2} } \\
& +(1-\sigma L / \delta)\left(1-\vartheta_{\tau(n)}\right) \alpha_{\tau(n)}\left(\frac{2}{1-\sigma L / \delta}\left\langle\sigma \phi\left(x^{\ddagger}\right)-\Psi\left(x^{\ddagger}\right), u_{\tau(n)}-\Psi\left(x^{\ddagger}\right)\right\rangle\right) .
\end{aligned}
$$

Since $\omega_{\tau(n)} \leqslant \omega_{\tau(n)+1}$, we have from (3.31) that

$$
\omega_{\tau(n)}^{2} \leqslant \frac{2}{1-\sigma L / \delta}\left\langle\sigma \phi\left(x^{\ddagger}\right)-\Psi\left(x^{\ddagger}\right), u_{\tau(n)}-\Psi\left(x^{\ddagger}\right)\right\rangle .
$$

Combining (3.31) with (3.32), we have

$$
\limsup _{n \rightarrow \infty} \omega_{\tau(n)} \leqslant 0
$$

and hence

$$
\lim _{n \rightarrow \infty} \omega_{\tau(n)}=0
$$

From (3.30) and (3.31), we also obtain

$$
\limsup _{n \rightarrow \infty} \omega_{\mathcal{\tau}(n)+1} \leqslant \limsup _{n \rightarrow \infty} \omega_{\mathcal{\tau}(n)} .
$$


This together with (3.33) implies that

$$
\lim _{n \rightarrow \infty} \omega_{\tau(n)+1}=0
$$

Applying Lemma 2.9 to get

$$
0 \leqslant \omega_{n} \leqslant \max \left\{\omega_{\tau(n)}, \omega_{\tau(n)+1}\right\} .
$$

Therefore, $\omega_{n} \rightarrow 0$. That is, $x_{n} \rightarrow \chi^{\ddagger}$. This completes the proof.

Algorithm 3.4. For given initial guess $x_{0} \in \mathcal{C}$ arbitrarily, let the sequence $\left\{x_{n}\right\}$ be generated iteratively by

$$
\left\{\begin{array}{l}
u_{n}=\operatorname{proj}_{\mathcal{C}}\left[\alpha_{n} \sigma \phi\left(x_{n}\right)+\left(1-\alpha_{n}\right)\left(x_{n}-\gamma_{n} \mathcal{B} x_{n}\right)\right], \\
x_{n+1}=\vartheta_{n} x_{n}+\left(1-\vartheta_{n}\right)\left[\left(1-\sigma_{n}\right) u_{n}+\sigma_{n} \mathcal{T}\left(\left(1-\delta_{n}\right) u_{n}+\delta_{n} \mathcal{T} u_{n}\right)\right], n \geqslant 0,
\end{array}\right.
$$

where $\phi: \mathcal{C} \rightarrow \mathcal{H}$ is an L-Lipschitz continuous mapping, $\left\{\alpha_{n}\right\},\left\{\vartheta_{n}\right\},\left\{\sigma_{n}\right\}$, and $\left\{\delta_{n}\right\}$ are four real number sequences in $[0,1],\left\{\gamma_{n}\right\}$ is a real number sequence in $(0, \infty)$, and $\sigma>0$ is a constant.

Corollary 3.5. Let $\mathcal{C}$ be a nonempty closed convex subset of a real Hilbert space $\mathcal{H}$. Let $\mathcal{B}: \mathcal{C} \rightarrow \mathcal{H}$ be an $\alpha$-inverse strongly monotone mapping. Let $\mathcal{T}: \mathcal{C} \rightarrow \mathcal{C}$ be a $\mathrm{k}$-Lipschitzian pseudocontractive operator with $\mathrm{K} \geqslant 1$. Suppose $\operatorname{VI}(\mathcal{B}, \mathcal{C}) \cap \operatorname{Fix}(\mathcal{T}) \neq \emptyset$. Assume the following conditions are satisfied:

(C1): $\lim _{n \rightarrow \infty} \alpha_{n}=0$ and $\sum_{n} \alpha_{n}=\infty$;

(C2): $0<\liminf _{n \rightarrow \infty} \vartheta_{n} \leqslant \lim \sup _{n \rightarrow \infty} \vartheta_{n}<1$ and $0<a<\sigma_{n}<c<\delta_{n}<b<\frac{1}{\sqrt{1+\kappa^{2}}+1}$;

(C3): $0<\liminf _{n \rightarrow \infty} \gamma_{n} \leqslant \lim \sup _{n \rightarrow \infty} \gamma_{n}<2 \alpha$.

Then, the sequence $\left\{x_{n}\right\}$ generated by (3.34) converges strongly to $x^{\ddagger} \in \mathrm{VI}(\mathcal{B}, \mathcal{C}) \cap \operatorname{Fix}(\mathcal{T})$ which solves the following variational inequality

$$
\left\langle\sigma \phi\left(x^{\ddagger}\right)-x^{\ddagger}, x^{b}-x^{\ddagger}\right\rangle \leqslant 0, \quad \forall x^{b} \in \operatorname{VI}(\mathcal{B}, \mathcal{C}) \bigcap \operatorname{Fix}(\mathcal{T}) .
$$

\section{Acknowledgment}

This research was partially supported by the Grants MOST 105-2115-M-039-002-MY3 and MOST 1062923-E-039-001-MY3.

\section{References}

[1] M. Aslam Noor, Some developments in general variational inequalities, Appl. Math. Comput., 152 (2004), 199-277. 1

[2] C. Byrne, A unified treatment of some iterative algorithms in signal processing and image reconstruction, Inverse Problems, 20 (2004), 103-120. 1

[3] L.-C. Ceng, Q. H. Ansari, J.-C. Yao, An extragradient method for solving split feasibility and fixed point problems, Comput. Math. Appl., 64 (2012), 633-642. 1

[4] L.-C. Ceng, Q. H. Ansari, J.-C. Yao, Relaxed extragradient methods for finding minimum-norm solutions of the split feasibility problem, Nonlinear Anal., 75 (2012), 2116-2125. 1

[5] Y. Censor, T. Bortfeld, B. Martin, A. Trofimov, A unified approach for inversion problems in intensity-modulated radiation therapy, Phys. Med. Biol., 51 (2006), 2353-2365. 1

[6] Y. Censor, T. Elfving, A multiprojection algorithm using Bregman projections in a product space, Numer. Algorithms, 8 (1994), 221-239.

[7] Y. Censor, T. Elfving, N. Kopf, T. Bortfeld, The multiple-sets split feasibility problem and its applications for inverse problems, Inverse Problems, 21 (2005), 2071-2084. 1

[8] F. Facchinei, J.-S. Pang, Finite-dimensional variational inequalities and complementarity problems, Vol. I, II, Springer Series in Operations Research, Springer-Verlag, New York, (2003). 1

[9] R. Glowinski, Numerical methods for nonlinear variational problems, Springer Series in Computational Physics, Springer-Verlag, New York, (1984). 1

[10] Z.-H. He, W.-S. Du, On hybrid split problem and its nonlinear algorithms, Fixed Point Theory Appl., 2013 (2013), 20 pages. 1 
[11] Z.-H. He, W.-S. Du, On split common solution problems: new nonlinear feasible algorithms, strong convergence results and their applications, Fixed Point Theory Appl., 2014 (2014), 16 pages. 1

[12] A. N. Iusem, An iterative algorithm for the variational inequality problem, Mat. Apl. Comput., 13 (1994), 103-114. 1

[13] G. M. Korpelevič, An extragradient method for finding saddle points and for other problems, Russian) Ékonom. i Mat. Metody, 12 (1976), 747-756. 1

[14] P. E. Maingé, Approximation methods for common fixed points of nonexpansive mappings in Hilbert spaces, J. Math. Anal. Appl., 325 (2007), 469-479. 2.9

[15] B. Qu, N.-H. Xiu, A note on the CQ algorithm for the split feasibility problem, Inverse Problems, 21 (2005), $1655-1665$. 1

[16] H.-K. Xu, Iterative algorithms for nonlinear operators, J. London Math. Soc., 66 (2002), 240-256. 2.8

[17] H.-K. Xu, A variable Krasnoselskiü-Mann algorithm and the multiple-set split feasibility problem, Inverse Problems, 22 (2006), 2021-2034. 1

[18] H.-K. Xu, Iterative methods for the split feasibility problem in infinite-dimensional Hilbert spaces, Inverse Problems, 26 (2010), 17 pages. 1

[19] Q.-Z. Yang, The relaxed CQ algorithm solving the split feasibility problem, Inverse Problems, 20 (2004), 1261-1266. 1

[20] Y.-H. Yao, R.-D. Chen, H.-K. Xu, Schemes for finding minimum-norm solutions of variational inequalities, Nonlinear Anal., 72 (2010), 3447-3456. 1

[21] Y.-H. Yao, W. Jigang, Y.-C. Liou, Regularized methods for the split feasibility problem, Abstr. Appl. Anal., 2012 (2012), 13 pages. 1

[22] Y.-H. Yao, Y.-C. Liou, S. M. Kang, Approach to common elements of variational inequality problems and fixed point problems via a relaxed extragradient method, Comput. Math. Appl., 59 (2010), 3472-3480. 1

[23] Y.-H. Yao, Y.-C. Liou, J.-C. Yao, Split common fixed point problem for two quasi-pseudo-contractive operators and its algorithm construction, Fixed Point Theory Appl., 2015 (2015), 19 pages. 1, 2.4, 2.5, 2.7

[24] Y.-H. Yao, M. A. Noor, Y.-C. Liou, Strong convergence of a modified extragradient method to the minimum-norm solution of variational inequalities, Abstr. Appl. Anal., 2012 (2012), 9 pages. 1

[25] Y.-H. Yao, M. A. Noor, Y.-C. Liou, S. M. Kang, Iterative algorithms for general multivalued variational inequalities, Abstr. Appl. Anal., 2012 (2012), 10 pages. 1

[26] Y.-H. Yao, M. Postolache, Y.-C. Liou, Strong convergence of a self-adaptive method for the split feasibility problem, Fixed Point Theory Appl., 2013 (2013), 12 pages. 1

[27] Y.-H. Yao, M. Postolache, Y.-C. Liou, Z.-S. Yao, Construction algorithms for a class of monotone variational inequalities, Optim. Lett., 10 (2016), 1519-1528. 1

[28] H. Zegeye, N. Shahzad, Y.-H. Yao, Minimum-norm solution of variational inequality and fixed point problem in Banach spaces, Optimization, 64 (2015), 453-471. 1

[29] L. J. Zhang, J. M. Chen, Z. B. Hou, Viscosity approximation methods for nonexpansive mappings and generalized variational inequalities, (Chinese) Acta Math. Sinica (Chin. Ser.), 53 (2010), 691-698. 1, 3

[30] J.-L. Zhao, Q.-Z. Yang, Several solution methods for the split feasibility problem, Inverse Problems, 21 (2005), 1791-1799. 1

[31] H.-Y. Zhou, Strong convergence of an explicit iterative algorithm for continuous pseudo-contractions in Banach spaces, Nonlinear Anal., 70 (2009), 4039-4046. 2.6 